\title{
The wide clinical expression spectrum presented in two families with NR5A 1 mutation
}

Context: Patients with NR5A1 mutations present a wide spectrum of phenotype. The gene is frequently associated with $46, \mathrm{XY}$ disorders of sex development (DSD).

Objectives: To analyze the clinical expression and NR5A1 mutations in two families with probands presenting 46, XY DSD.

Results: Two probands with 46, XY gonadal dysgenesis from two unconsanguineous families showed spontaneous puberty, normal levels of ACTH, cortisol, testosterone, increased FSH and decreased LH/FSH, AMH and INHB. In Family 1, a novel pathogenic variant, p.C33X, was identified in the proband. By determining other members in this family, the variant was found in the proband's mother, two elder sisters and two nephews. However, no obvious anomaly was presented in the eldest sister with the variant while her two sons showed hypospadias. In Family 2, the proband inherited a reported pathogenic variant, p.M1I from his mother who delivered 4 children by natural pregnancy. The self-reported family puberty and fertility history was negative until we calculated the exact menopause age of mothers at 36 and 41 years of age respectively.

Conclusion: The 46, XY DSD patients with NR5A1variants show highly variable phenotypes, from complete female genitalia to ambiguous external genitals/hypospadias. While $46, X X$ individual may have inconspicuous symptoms. Normal fertility is not a reliable negative family history on this disease. The identified novel pathogenic variant, p.C33X in patient 46, XY DSD and 46, XX primary ovarian insufficiency extends the genotypic and phenotypic spectrum of NR5A1. This study also highlights the critical role of NR5A1 protein in gonadal development and differentiation.

\section{Keywords: NR5A1gene, pathogenic variant, phenotype, gonadal dysgenesis}

\section{Introduction}

Disorders of sex development (DSDs) are a group of heterogeneous diseases characterized by a rare set of conditions in which the chromosomal, gonadal, and phenotypic sex is atypical [1]. Nuclear receptor subfamily 5 group A member 1 (NR5A1, OMIM184757) plays a crucial role in regulating adrenal development, gonad determination and differentiation [2]. NR5A1 gene maps on $9 \mathrm{q} 33$ and has one non-translated exon (exon 1) and six other coding exons (exon 2-7) [3]. It encodes the steroidogenic factor 1(SF-1) consisting of 461 amino acid residuals that regulate several steps of adrenal and gonadal development at early stages of embryonic development. The SF1 has two zinc-finger DNA-binding domains (DBD), a ligand-binding domain (LBD), two functional activation domains (AF-1 and AF-2), an accessory region and a hinge region [4].The
DBD contains a core with two Cys4-zinc-finger motifs and a highly conserved Ftz-F1 box motif potentially involved in interaction with DNA [5]. NR5A1 is highly conserved among different species, and the homology of mice is up to $95 \%$ of human NR5A1 gene [6].

In 46, XY individuals, SF-1 activates the expression of anti-Müllerian hormone (AMH) in Sertoli cells, resulting in the regression of Müllerian structures, and inducing production of steroidogenic enzymes in Leydig cells, facilitating the virilzation of external genitalia and testes descending [7]. The protein functions in 46, XX individuals mainly as a promotor for follicle development and maturation. Therefore, individuals with $N R 5 A 1$ mutation are mainly characterized by $46, \mathrm{XY}$ DSD or $46, \mathrm{XX}$ premature ovarian failure $[23,25,27,28]$.

Achermann [8,9] reported initially in 1999 and 2002 that heterozygous mutations of
Yanning Song ${ }^{1,2}$, LijunFan $^{1,2}$, Xiu Zhao ${ }^{2}$, Xiaoya Ren ${ }^{1,2}$, Beibei Zhang ${ }^{1,2}$ \& Chunxiu Gong ${ }^{* 1,2,3}$ ${ }^{1}$ National Center for Children's Health, China Center of Children's Health, Beijing Children's Hospital affiliated to Capital Medical University, PR China

${ }^{2}$ Department of Endocrinology, Genetics, Metabolism and Adolescent Medicine, Beijing Children's Hospital, Capital Medical University,56\# Nan Lishi Rd, West District, Beijing, PR China

${ }^{3}$ Beijing Key Laboratory for Genetics of Birth Defects, Beijing Children's Hospital, Capital Medical University, 56\# Nan Lishi Rd, West District, Beijing, PR China

*Author for correspondence: chunxiugong@vip.163.com 
NR5A1 gene could cause adrenal insufficiency and 46, XY severe testicular dysplasia. Then the following researches found heterozygous NR5AI mutations could cause ambiguous genitalia, gonadal dysgenesis without adrenal insufficiency in 46, XY patient [10-14]. Further, Achermann [9] studied a consanguineous pedigree and found that the proband with the homozygous mutation (R92Q) of NR5A1 showed adrenal insufficiency and gonadal dysgenesis while the heterozygous parents and sister expressed no significant clinical phenotype, which is consistent with the results presented by Sadovsky et al. [15] who found that homozygous mutation of $\mathrm{Nr} 5 \mathrm{al}$ in mice could have profound adrenal insufficiency and gondal development, while heterozygous mutation only cause gondal dysgenesis. So, they hypothesized that the SF-1function in developmental pathways in humans is dosage sensitive. Most recently, case reports indicated that NR5A1 mutations can be associated with a wide spectrum of phenotypes, including 46,XX premature ovarian failure and even sex reverse like testicular DSD or ovotesticular DSD [16-21].The relationship between genotype and phenotype has not been established yet because of relatively rare cases and the reported phenomenon of incomplete penetrance $[11,23]$.In this study, the patients with $N R 5 A 1$ mutations from two unrelated families showed widely variable phenotype in 46,XY individuals and impairment of ovarian function in 46,XX individuals without evidence of adrenal failure.

\section{Subjects and methods \\ - Subjects}

Two probands of 46, XY DSD and their family member were recruited in this study and informed consent was obtained. All the family members declined to take more clinical examinations except genetic testing.

\section{Methods}

Clinical information of the two probands were collected, including family history, physical examination, serum electrolyte and thyroid function, Adrenocorticotropic hormone (ACTH), Cortisol (Cor), Testosterone (T), Dihydrotestosterone (DHT), Anti Müllerian Hormone (AMH), Inhibin B (INHB), pelvic and testicular ultrasound etc. For genetic testing of the subjects, the AidLab DN01 kit was used to extract the genomic DNA from $2 \mathrm{ml}$ of the peripheral blood in the EDTA anticoagulant tube. According to the primers and PCR reaction conditions as reported [22], all the exons were amplified. The PCR products were detected by $2 \%$ agarose gel electrophoresis and were sequenced. The obtained sequences were aligned and mapped to the reference of GeneBank (NM_004959.4). Clustal Omega (http:/ / www. ebi. AC. uk/ Tools/ msa/clustalo/) was used to analyze the conservation of the novel variant.

\section{Results \\ - Clinical features}

The proband 1 was a social girl (Subject II-11) presented at the age of 17 years old with primary amenorrhea and virilized for 4 years. When she was born, normal genitals were presented, and the height and weight were in normal as female peers. At age of 13, she showed hoarseness, clitoris hypertrophy but no breast development. As her mother had breast development until 16 years old, so no attention was paid on. Till 17 years old, she got a karyotyping result of $46, \mathrm{XY}$ at a local hospital, and then was referred to our hospital. She was boyish and always played with boys since very young school age according to her mother's description. Physical examination showed normal blood pressure $(110 / 60 \mathrm{mmHg})$, armpit hair sparse, bilateral breast Tanner 1 and the clitoris enlargement like the penis (length of $5.6 \mathrm{~cm}$ ). The urethra was open at the perineum and blind vagina. Her mother (Subject I-2) had menarche at 17 years old, natural pregnancy at 21 and was menopause at 36 years.SubjectII-3 and subjectII-7died a few days after birth because of unknown reasons. The age of subjectII-1, II4, II-5, II-8, II-9, II-10 were 31, 30, 29, 26, 22 , 21years old and the menarche were 16-17 years old, respectively. Both subjectIII-1 and subject III-2 had hypospadias and had been repaired.

The proband 2 (Subject II-4) came from an unconsanguineous family, presenting with signs of virilization at the age of 10.5 years. She presented at birth with ambiguous external genitalia and was reared as female. Recently, she presented mustache, voice deepen and external genitalia changes. Physical examination showed the heightwas $148 \mathrm{~cm}$ and the weight was $50 \mathrm{~kg}$. The bilateral breast was in Tanner 1 with apenislike3-cm long clitoris. The urethra was open at the perineum (FIGURE 1). The karyotype was 46, XY. The mother of the proband 2(Subject I-2) had regular menstrual and natural pregnancy at the age of 23 years and was menopause at 41 
years. SubjectII-1 presented with ambiguous sex external genitalia and died at 3 month with unknown reason. Subject II-2, II-3 were miscarried because of unknown reasons. SubjectII-5 was a normal boy. The electrolyte and thyroid function of the two probands were normal. The B-ultrasound examination of renal and adrenal gland was normal. Clinical features of the two families were shown in TABLE 1.

\section{Sequence analysis of the NR5A1 gene}

A heterozygous nonsense variant, c.99C >A, p.C33X (FIGURE 2A) was identified in Proband1, subjectI-2, II-1, II-3 and III-1, III-2. This mutation is predicted to alter the protein sequence and create a premature termination, that is c.99C $>\mathrm{A}$ coding the p. C33X then leading to deletion of 228 amino acids in SF-1 protein. The multiple species alignment suggested that cysteine on this site and the following 228 amino acid residuals were highly conservative (FIGURE 2B). The variant was not found in HGMD and ExAC database. Proband 2 and her mother had a heterozygous variant, c.3G>A, p.M1I (FIGURE 2A), which has been identified as pathogenic variation in HGMD and clinvar database. This variant is located at the initiation codon, which destroys the initiation of translation, leading to protein translation defects [23].

Proband1 had been operated being a boy by his own decision after understanding the situation and had repaired hypospadias in August. Proband2 was 10 years old and

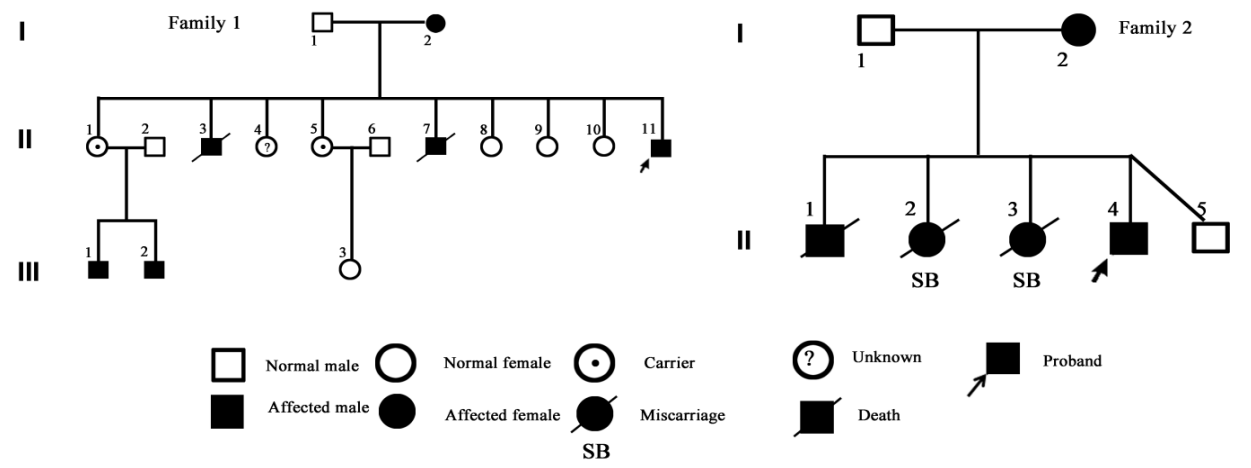

FIGURE 1. Pedigrees of the two Families with 46, XY DSD.

Subject I-1, II-4 in family 1 were declined to do gene test and subject III-3 was a normal girl.

Subject II-4 and II-5 were biovular twins.p

A
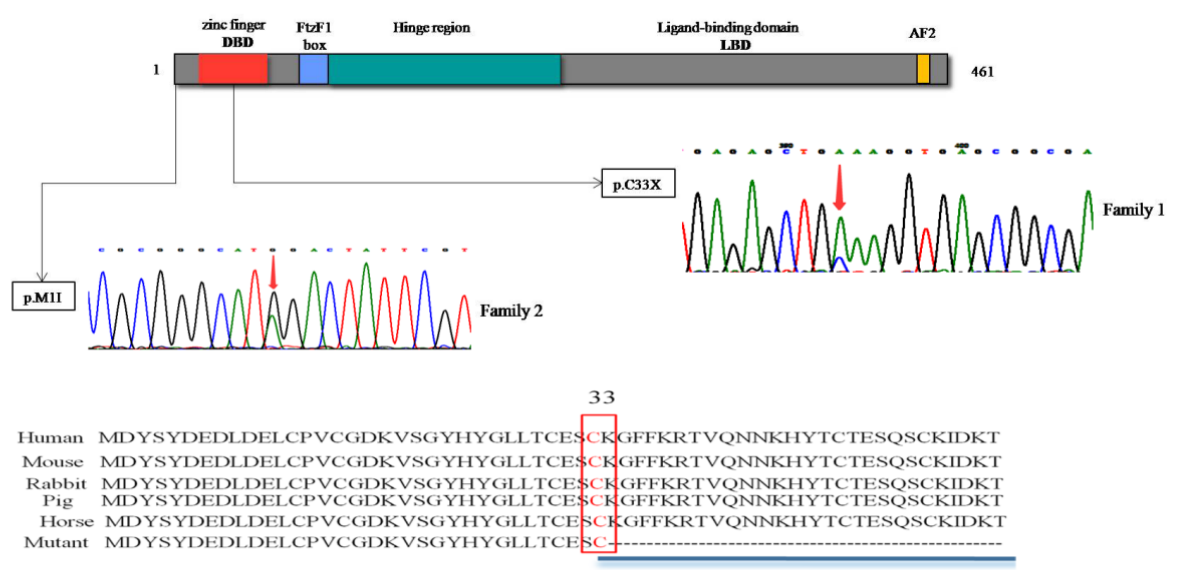

FIGURE 2. Distribution of NR5A1 Mutations in Relation to the Protein.

A: The functional domains of the NR5A1 protein are shown, indicating the position of known NR5A1 mutations, The DNA-binding domain (DBD) containing two zinc-finger motifs is indicated. The FtzF1 box stabilizes protein binding to DNA. The hinge region is important for stabilizing the ligand-binding domain and interacts with other proteins that control NR5A1 transcriptional activity. The AF2 domain recruits cofactors necessary for NR5A1 transactivation activity

B: Showed the NR5A1 mutation of family 1 was highly conservative in difference species. 


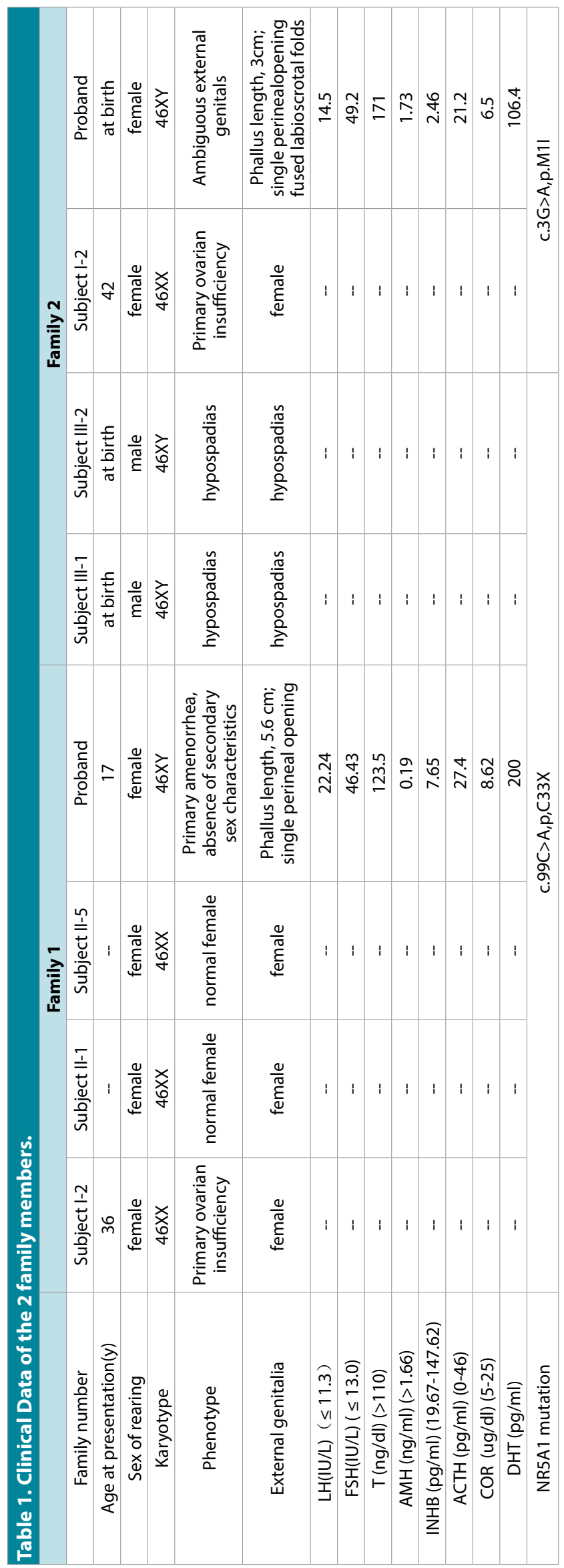


hesitant whether to continue being a girl or a boy, So GnRHa was used to inhibit puberty development until mental maturity.

\section{Discussion}

Widely variable of NR5A1-related phenotypes were well documented in $46, \mathrm{XY}$ patients [17,24-27]. And the spectrum of disorders associated with NR5A1 variants in 46, XX individuals was further expanded by the demonstration of the specific recurrent heterozygous p.Arg92Trp NR5A1 variant in association with variable degrees of testis development in 46, XX patients from unrelated families [20]. In this research, the proband1 presented with complete female genitalia at birth and appeared masculinization in adolescence. Her two brothers died several days after birth of unknown causes. It is difficult to rule out whether NR5A1 mutation leading to adrenal insufficiency that adrenal crisis causing death. Two nephews of the proband 1 carried the variant showed different degrees of hypospadias. These results indicated that regardless of the same variant in the family, different $46, \mathrm{XY}$ individuals may present different phenotypes.

The two probands mothers showed premature ovarian failure (POF) and earlier menopause, suggesting that screening NR5A1 gene is necessary in POF patients, as proposed in other research [28].However, due to the variable phenotypes and incomplete penetrance, some female carriers may be easily misdiagnosed because of normal fertility preserved in $46, \mathrm{XX}$ individual, as shown in the two mothers and the II-1 of family 1 . Thus, negative family history, normal fertility, and normal puberty are not always reliable criteria. These features in the two families made us uncertain at the beginning until we got their menopause ages. Moreover, the unreliable negative family history may come from concealment of the mother due to pressure from the family. For the sisters who carried the same mutant allele, we should pay more attention to signs of POF as indicated from their mother. Regular follow-up, evaluation and appropriate treatments should be taken when necessary.

After the gonadal differentiation into testis of a 46, XY individual, SF-1 was expressed in the early testes continuously, maintaining the expression of SOX 9 together with SRY. In 7 weeks of pregnancy, SF-1 activates the expression of $\mathrm{AMH}$, which induces the degeneration of the Müllerian structure and activates the steroid enzyme synthesis to maintain the development of masculine external genitals from the stage of 8 weeks [29]. The damage of leydig cells at the early stage of embryonic development caused by pathogenic NR5Almutations can lead to inadequate secretion of $\mathrm{T}$; therefore, patients with $N R 5 A 1$ mutations always present deficiency in virilization and are reared as female in most cases. However, at puberty they show hoarseness, prominent Adam's apple and hypertrophy of the clitoris [31,32], similar situations as indicated in the probands of this study.

Most patients have no remnants of Müllerian structure attributing to sufficient AMH produced at the early stage of embryonic development to degenerate the Müllerian structure. Previous research found the function of Storli cells gradually decreases with age [30], which can be inferred from the patients in this study. Absence of uterus in the two probands indicated AMH secretion by Storli cells were enough during embryonic development. Elevated levels of both LH and FSH with expected low level of suggested a condition of hypergonadotropic hypogonadism. A few studies have confirmed that 46, XY patients with NR5A1 mutation can present spontaneous puberty [31,32] and continuous elevation of FSH level in the followup, suggesting damage of Storli cells and the resulting dyszoospermia. This also explains why rare males with $N R 5 A 1$ mutation have fertility function [33-35]. For the two probands in our study, we observed that $\mathrm{LH} / \mathrm{FSH}$ ratio, AMH and INHB levels decreased significantly with a normal level of testosterone, indicating the damage of Storli cells may be more severely than that of Leydig cells. Both the two cases showed spontaneous puberty and virilization probably induced by the residual cells, consistent with the former research [31-32].

To date, nearly 120 NR5A1 variants have been documented in Human Gene Mutation Database (http:// www.hgmd.cf.ac.uk/ac/gene. php?gene $=N R 5 A 1)$, including nonsense variants (the most common), missense variants, small deletions and duplications, small indels, and splicing variants. The genotype-phenotype relationship is unclear due to deficiency of studies of phenotypic diversity with large content of human cases. In this study, we identified a 
novel heterozygous nonsense variant (c.99C $>\mathrm{A}$, p.C33X) in family 1 and a known pathogenic variant (c.3G>A, p.M1I) in the second family.

Generally, mutations in the DBD region of NR5A1 directly interacts with DNA and may alter patterns or recognition of specific DNA sequences, resulting in reduced expression of downstream target genes and disruption of gonadal development regulation. The variant, p.C33X is located in DBD region, directly combined with the first zinc finger protein. It is known that cysteine affects the 3D structure of the whole protein. Its sulfenyl reacts with other groups to form disulfide bond, maintaining protein folding and stability. Cysteine residues also play an important role in cross-linked proteins, which increase the rigidity of protein, and increase the resistance to hydrolysis. Therefore, cysteine changes are not only lead to the early termination of the protein translation, but also lose the ability of DNA to combine with the zinc finger, causing loss of the whole protein function. Although no functional verification was seen, multiple sequence comparisons indicated that the amino acid in this locus was conservative. Combined with family information, clinical symptoms and the nature of this mutation (nonsense mutation), we identified the novel mutation as pathogenic.

The c.3G $>\mathrm{A}$ p.M3I was first reported in a family by Lourenco et al. [23] in 2009. Both the cases (46, XX girls), carrying a heterozygous mutation (c.3G>A p.M3I), presented a partial gonadal dysgenesis and primary ovarian insufficiency respectively (irregular menstruation, menstruation ended at 15 years old). Their mother carrying the same mutation without phenotype was interpreted as incomplete penetrance [11]. Translation initiation at this codon is predicted to truncate the N-terminal of the protein, eliminating the DNA-binding domain.
DSD sex rearing has been a hot topic of debate. Many masculine females with NR5A1 variants chose to change gender [25,31]. As showed above, the boys with NR5A1 mutations presented spontaneous puberty, and other similar patients with preserved fertility had been reported [31,32], hinting certain advantages in rearing those 46, XY patients with NR5A1 mutation as boys. In this study, proband 1 has taken repair operation for hypospadia and proband 2 has been waiting until old enough to make further evaluation.

\section{Conclusion}

In this study, we identified a novel nonsense variant and a known pathogenic variant of NR5A1 gene from two unrelated families. The 46, XY DSD patients with NR5A1 variants show highly variable phenotypes, from complete female genitalia to ambiguous external genitals/hypospadias. The 46, XX individuals with NR5Alvariants could be misdiagnosed due to atypical clinical signs. The identified novel pathogenic variant and detailed clinical characteristics in patients extend the genotypic and phenotypic spectrum of NR5A1. Screening of NRSA1 gene could improve diagnosis of DSD.

\section{Conflict of interest statement}

All authors declare that no conflicts of interest exist regarding this work.

\section{Acknowledgments}

This work was funded by the Public Health Project for Residents in Beijing (Z151100003915103) and the National Key Research and Development Programme of China (2016YFC0901505).We thank Rui Wang for her help with blood collection and the researchers at the Beijing Key Laboratory for Genetics of Birth Defects for the gene pathogenicity analysis. 


\section{REFERENCES}

Hughes IA, Houk C, Ahmed SF, Lee PA. Consensus statement on management of intersex disorders. J. Pediatr. Urol. 2(3), 148-162 (2006).

Gerster K, Biason-Lauber A, Schoenle EJ. Clinical follow-up of the first SF-1 insufficient female patient. Ann. Endocrinol. (Paris) 78(3), 156-161 (2017).

Taketo M, Parker KL, Howard TA, et al. Homologs of Drosophila Fushi-Tarazu factor 1 map to mouse chromosome 2 and human chromosome 9q33. Genomics. 25(2), 565-567 (1995).

Parker KL, Schimmer BP. Steroidogenic factor 1: a key determinant of endocrine development and function. Endocr. Rev. 18(3), 361-377 (1997).

Venselaar H, Te BT, Kuipers RK, Hekkelman ML, Vriend G. Protein structure analysis of mutations causing inheritable diseases. An e-Science approach with life scientist friendly interfaces. $B M C$ Bioinformatics. 11, 548 (2010).

Ohno CK, Petkovich M. FTZ-F1 beta, a novel member of the Drosophila nuclear receptor family. Mech. Dev. 40(12), 13-24 (1993).

Buaas FW, Gardiner JR, Clayton S, Val P, Swain A. In vivo evidence for the crucial role of SF1 in steroid-producing cells of the testis, ovary and adrenal gland. Development. 139(24), 4561-4570 (2012).

Achermann JC, Ito $M$, Ito $M$, Hindmarsh PC, Jameson JL. A mutation in the gene encoding steroidogenic factor- 1 causes XY sex reversal and adrenal failure in humans. Nat. Genet. 22(2), 125126 (1999).

Achermann JC, Ozisik G, Ito M, et al. Gonadal determination and adrenal development are regulated by the orphan nuclear receptor steroidogenic factor-1, in a dose-dependent manner. J. Clin. Endocrinol. Metab. 87(4), 1829-1833 (2002).

Mallet D, Bretones P, MichelCalemard L, et al. Gonadal dysgenesis without adrenal insufficiency in a 46, XY patient heterozygous for the nonsense C16X mutation: a case of SF1 haploinsufficiency. J. Clin. Endocrinol. Metab. 89(10), 4829-4832 (2004).

Philibert P, Zenaty D, Lin L, et al. Mutational analysis of steroidogenic factor 1 (NR5a1) in 24 boys with bilateral anorchia: a French collaborative study. Hum. Reprod. 22(12), 3255-3261 (2007).

Kohler B, Lin L, Mazen I, et al. The spectrum of phenotypes associated with mutations in steroidogenic factor 1 (SF1, NR5A1, Ad4BP) includes severe penoscrotal hypospadias in 46, XY males without adrenal insufficiency. Eur. J. Endocrinol. 161(2), 237-242 (2009).

Tajima T, Fujiwara F, Fujieda K. A novel heterozygous mutation of steroidogenic factor-1 (SF-1/Ad4BP) gene (NR5A1) in a 46, XY disorders of sex development (DSD) patient without adrenal failure. Endocr. J. 56(4), 619-624 (2009).

Kohler B, Lin L, Ferraz-de-Souza B, et al. Five novel mutations in steroidogenic factor 1 (SF1, NR5A1) in 46,XY patients with severe underandrogenization but without adrenal insufficiency. Hum. Mutat. 29(1), 59-64 (2008).

Sadovsky Y, Crawford PA, Woodson $\mathrm{KG}$, et al. Mice deficient in the orphan receptor steroidogenic factor 1 lack adrenal glands and gonads but express P450 sidechain-cleavage enzyme in the placenta and have normal embryonic serum levels of corticosteroids. Proc. Natl. Acad. Sci. USA. 92(24), 10939-10943 (1995).

Allali S, Muller JB, Brauner R, et al. Mutation analysis of NR5A1 encoding steroidogenic factor 1 in 77 patients with 46, XY disorders of sex development (DSD) including hypospadias. Plos ONE. 6(10), e24117 (2011).

Domenice S, Machado AZ, Ferreira FM, Ferraz-de-Souza B, Lerario AM, Lin L, et al. Wide spectrum of NR5A1related phenotypes in 46,XY and 46,XX individuals. Birth Defects Res. C. Embryo Today. 108(4), 309-320 (2016).

Swartz JM, Ciarlo R, Guo MH, et al. A 46, XX Ovotesticular Disorder of Sex Development Likely Caused by a Steroidogenic Factor-1 (NR5A1) Variant. Horm. Res. Paediatr. 87(3), 191-195
(2017).

Miyado $M$, Inui $M$, Igarashi $M$, et al. The p.R92W variant of NR5A1/ $\mathrm{Nr} 5 \mathrm{a} 1$ induces testicular development of 46,XX gonads in humans, but not in mice: phenotypic comparison of human patients and mutation-induced mice. Biol. Sex Differ. 7, 56 (2016).

Baetens D, Stoop H, Peelman F, Todeschini AL, Rosseel T, Coppieters F, et al. NR5A1 is a novel disease gene for 46,XX testicular and ovotesticular disorders of sex development. Genet. Med. 19(4), 367-376 (2017).

Igarashi M, Takasawa K, Hakoda A, et al. Identical NR5A1 Missense Mutations in Two Unrelated 46,XX Individuals with Testicular Tissues. Hum. Mutat. 38(1), 39-42 (2017).

Robevska G, van den Bergen JA, Ohnesorg $T$, et al. Functional characterization of novel NR5A1 variants reveals multiple complex roles in disorders of sex development. Hum. Mutat. 39(1), 124-139 (2018).

Lourenco D, Brauner R, Lin L, et al. Mutations in NR5A1 associated with ovarian insufficiency. N. Engl. J. Med. 360(12), 1200-1210 (2009).

Warman DM, Costanzo M, Marino $\mathrm{R}$, et al. Three new SF-1 (NR5A1) gene mutations in two unrelated families with multiple affected members: within-family variability in $46, \mathrm{XY}$ subjects and low ovarian reserve in fertile $46, \mathrm{XX}$ subjects. Horm. Res. Paediatr. 75(1), 70-77 (2011).

Camats N, Pandey AV, FernandezCancio $\mathrm{M}$, et al. Ten novel mutations in the NR5A1 gene cause disordered sex development in 46,XY and ovarian insufficiency in 46,XX individuals. J. Clin. Endocrinol. Metab. 97(7), E1294-1306 (2012).

Lin L, Philibert P, Ferraz-de-Souza B, Kelberman D, Homfray T, Albanese A, et al. Heterozygous missense mutations in steroidogenic factor 1 (SF1/Ad4BP, NR5A1) are associated with 46,XY disorders of sex development with normal adrenal function. J. Clin. Endocrinol. Metab. 92(3), 991-999 (2007).

Reuter AL, Goji K, Bingham NC, 
Matsuo M, Parker KL. A novel mutation in the accessory DNA-binding domain of human steroidogenic factor 1 causes $\mathrm{XY}$ gonadal dysgenesis without adrenal insufficiency. Eur. J. Endocrinol. 157(2), 233-238 (2007).

Qin Y, Jiao X, Simpson JL, Chen ZJ. Genetics of primary ovarian insufficiency: new developments and opportunities. Hum. Reprod. Update. 21(6), 787-808 (2015).

Kato T, Esaki M, Matsuzawa A, Ikeda Y. NR5A1 is required for functional maturation of Sertoli cells during postnatal development. Reproduction. 143(5), 663-672 (2012).

Tantawy S, Mazen I, Soliman H, et al. Analysis of the gene coding for steroidogenic factor 1 (SF1, NR5A1) in a cohort of 50 Egyptian patients with $46, \mathrm{XY}$ disorders of sex development. Eur. J. Endocrinol. 170(5), 759-767 (2014).

Tantawy S, Lin L, Akkurt I, et al. Testosterone production during puberty in two 46,XY patients with disorders of sex development and novel NR5A1 (SF1) mutations. Eur. J. Endocrinol. 167(1), 125-130 (2012).

Fabbri HC, de Andrade JG, Soardi FC, et al. The novel p.Cys65Tyr mutation in NR5A1 gene in three 46 ,XY siblings with normal testosterone levels and their mother with primary ovarian insufficiency. BMC Med. Genet. 15, 7 (2014).
Bashamboo A, Ferraz-de-Souza B, Lourenco D, et al. Human male infertility associated with mutations in NR5A1 encoding steroidogenic factor 1. Am. J. Hum. Genet. 87(4), 505-512 (2010).

Philibert P, Polak M, Colmenares A, et al. Predominant Sertoli cell deficiency in a $46, \mathrm{XY}$ disorders of sex development patient with a new NR5A1/SF-1 mutation transmitted by his unaffected father. Fertil. Steril. 95(5), 1785-1788 (2011).

Yagi H, Takagi M, Kon M, et al. Fertility preservation in a family with a novel NR5A1 mutation. Endocr. J. 62(3), 289-295 (2015). 